\title{
Solving the backward heat equation on the unit sphere
}
Q. T. Le Gia ${ }^{1}$
Huy Tuan Nguyen ${ }^{2}$
Thanh $\operatorname{Tran}^{3}$

(Received 26 February 2015; revised 8 January 2016)

\begin{abstract}
We consider an inverse problem for the heat equation on the unit sphere in which the final (current) temperature on the sphere is given, and the task is to determine the initial temperature. The problem is ill-posed in the sense of Hadamard; hence, a regularization technique is applied. We then use a Galerkin method with spherical radial basis functions to solve the regularized problem. The problem may have potential applications in atmospheric modelling, when current temperature data is used to calculate a past global temperature.
\end{abstract}

http://journal.austms.org.au/ojs/index.php/ANZIAMJ/article/view/9346 gives this article, (c) Austral. Mathematical Soc. 2016. Published January 19, 2016, as part of the Proceedings of the 17th Biennial Computational Techniques and Applications Conference. ISSN 1446-8735. (Print two pages per sheet of paper.) Copies of this article must not be made otherwise available on the internet; instead link directly to this URL for this article. 


\section{Contents}

1 Introduction

C263

2 Preliminaries

C264

2.1 Spherical harmonics . . . . . . . . . . . . . C264

2.2 Positive definite kernels . . . . . . . . . . . . . . . C265

3 Ill-posedness of the backward heat equation on $\mathbb{S}^{n}$

C267

4 Regularization and approximation

C269

4.1 The regularized problem . . . . . . . . . . . . C269

4.2 Galerkin approximation to the regularized problem . . . C 270

5 Numerical experiments

C271

6 Conclusion

$\mathrm{C} 275$

References

C276

\section{Introduction}

Backward parabolic equations have applications in hydrologic inversion and image deblurring. In hydrologic inversion [1], sources of groundwater pollution are sought by reconstructing the contaminant history. Kovasznay and Joseph [6] proposed the use of a backward diffusion equation to restore blurred images. Backward parabolic problems on a spherical domain have potential applications in atmospheric modelling, when a global temperature from the past is to be determined by current temperature data. Enting [4] discussed more examples of inverse problems in atmospheric modelling.

Backward parabolic problems are ill-posed in the sense of Hadamard [5] (see Section 3). Hence, in practice, regularization techniques are used to 
transform the backward parabolic problem into a well-posed problem which can be solved. Section 3 discusses how to solve the regularized problem using spherical radial basis functions.

Let $\mathbb{S}^{n}$ be the unit Euclidean sphere in $\mathbb{R}^{n+1}$. The backward heat problem on $\mathbb{S}^{n}$ is: determine $u(x, t)$ in time interval $0 \leqslant t<T$ which satisfies

$$
\begin{cases}\frac{\partial}{\partial t} \mathfrak{u}(x, t)-\Delta^{*} \mathfrak{u}(x, t)=0, & 0<t<T, \\ u(x, T)=f(x), & x \in \mathbb{S}^{n} .\end{cases}
$$

Here, $\Delta^{*}$ denotes the Laplace-Beltrami operator on the unit sphere. Following a strategy for a more general parabolic problem proposed by Clark and Oppenheimer [3], we consider the regularized problem

$$
\begin{cases}\frac{\partial}{\partial t} u^{\epsilon}(x, t)-\Delta^{*} u^{\epsilon}(x, t)=0, & 0<t<T, \\ u^{\epsilon}(x, T)+\alpha(\epsilon) u^{\epsilon}(x, 0)=f^{\epsilon}(x), & x \in \mathbb{S}^{n} .\end{cases}
$$

Here $\alpha(\epsilon)$ is a regularization term which depends on some given parameter $\epsilon$ satisfying $0<\epsilon<1$, and $\boldsymbol{f}^{\epsilon}$ is a perturbed version of $f$ so that $\left\|f^{\epsilon}-f\right\|_{L^{2}\left(\mathbb{S}^{n}\right)}<$ $M \epsilon$ with some fixed constant $M$. Section 4 explains the idea behind the special choice of the regularization term in equation (2).

By shifting the time variable $t$, without loss of generality, we determine the solution at $t=0$ only. Section 4.1 converts the regularized problem at $\mathbf{t}=0$ into a pseudo-differential equation on $\mathbb{S}^{n}$. Section 4.2 solves the pseudo-differential equation with a Galerkin method using spherical radial basis functions.

\section{Preliminaries}

\subsection{Spherical harmonics}

A spherical harmonic is the restriction of a homogeneous harmonic polynomial in $\mathbb{R}^{n+1}$ to the unit sphere $\mathbb{S}^{n}$. The space of spherical harmonics of degree $\ell$, 
denoted by $\mathcal{H}_{\ell}$, has dimension

$$
\begin{aligned}
& N(n, 0)=1 \text { for } \ell \geqslant 1, \text { and } \\
& N(n, \ell)=\frac{(2 \ell+n-1)(\ell+n-2) !}{\ell !(n-1) !} \text { for } \ell \geqslant 1 .
\end{aligned}
$$

The space $\mathcal{H}_{\ell}$ has a natural orthonormal basis $\left\{\mathrm{Y}_{\ell, k}: 1 \leqslant k \leqslant N(n, \ell)\right\}$, where $Y_{\ell, k}$ is the spherical harmonic of degree $\ell$ and order $k$ [7]. Spherical harmonics with different degrees and different orders are orthogonal with each other, so

$$
\left\langle Y_{\ell, k}, Y_{\ell^{\prime}, k^{\prime}}\right\rangle:=\int_{\mathbb{S}^{n}} Y_{\ell, k} Y_{\ell, k^{\prime}} d S=\delta_{\ell, \ell^{\prime}} \delta_{k, k^{\prime}},
$$

where dS is the surface measure of $\mathbb{S}^{n}$ and $\delta_{k, k^{\prime}}$ is the Kronecker delta. Spherical harmonics are eigenfunctions of the Laplace-Beltrami operator $\Delta^{*}$ on $\mathbb{S}^{n}[7]$, that is, for $\ell=0,1,2, \ldots$ and $1 \leqslant k \leqslant N(n, \ell)$,

$$
-\Delta^{*} Y_{\ell, k}=\lambda_{\ell} Y_{\ell, k} \quad \text { where } \quad \lambda_{\ell}=\ell(\ell+n-1) .
$$

Every function $v \in \mathrm{L}^{2}\left(\mathbb{S}^{n}\right)$ is represented by a generalised Fourier series

$$
v=\sum_{\ell=0}^{\infty} \sum_{k=1}^{N(n, \ell)} \widehat{v}_{\ell, k} Y_{\ell, k} \quad \text { where } \quad \widehat{v}_{\ell, k}=\left\langle v, Y_{\ell, k}\right\rangle .
$$

\subsection{Positive definite kernels}

A continuous function $\Phi: \mathbb{S}^{n} \times \mathbb{S}^{n} \rightarrow \mathbb{R}$ is called a positive definite kernel [9] on $\mathbb{S}^{n}$ if it satisfies the two conditions

(i) $\Phi(\boldsymbol{x}, \mathbf{y})=\Phi(\mathbf{y}, \boldsymbol{x})$ for all $\boldsymbol{x}, \boldsymbol{y} \in \mathbb{S}^{n}$;

(ii) for any set of distinct scattered points $\left\{\boldsymbol{y}_{1}, \mathbf{y}_{2}, \ldots, \mathbf{y}_{K}\right\} \subset \mathbb{S}^{n}$, the symmetric $\mathrm{K} \times \mathrm{K}$ matrix $\left[\Phi\left(\boldsymbol{y}_{i}, \mathbf{y}_{j}\right)\right]$ is positive semi-definite. 
We call $\Phi$ strictly positive definite if the matrix $\left[\Phi\left(\mathbf{y}_{i}, \mathbf{y}_{j}\right)\right]$ is positive definite. We work with a kernel $\Phi$ defined in terms of a univariate function $\phi$ : $[-1,1] \rightarrow \mathbb{R}$ by

$$
\Phi(\boldsymbol{x}, \mathbf{y})=\phi(\boldsymbol{x} \cdot \mathbf{y}) \quad \text { for all } \boldsymbol{x}, \boldsymbol{y} \in \mathbb{S}^{n}
$$

where $\boldsymbol{x} \cdot \boldsymbol{y}$ denotes the Euclidean scalar product of $\boldsymbol{x}$ and $\mathbf{y}$. Following Müller [7], let $P_{\ell}(t)$ denote the Legendre polynomial of degree $\ell$, and expand $\phi(t)$ in a Fourier-Legendre series

$$
\phi(t)=\frac{1}{\omega_{n}} \sum_{\ell=0}^{\infty} N(n, \ell) \widehat{\phi}(\ell) P_{\ell}(t),
$$

in which

$$
\widehat{\phi}(\ell)=\omega_{n-1} \int_{-1}^{1} \phi(t) P_{\ell}(t)\left(1-t^{2}\right)^{(n-2) / 2} d t,
$$

where $\omega_{n}$ is the surface area of $\mathbb{S}^{n}$. Using the addition formula for spherical harmonics [7, p. 10],

$$
\sum_{k=1}^{N(n, \ell)} Y_{\ell, k}(\boldsymbol{x}) Y_{\ell, k}(y)=\frac{N(n, \ell)}{\omega_{n}} P_{\ell}(\boldsymbol{x} \cdot \mathbf{y})
$$

the kernel is

$$
\Phi(\boldsymbol{x}, \mathbf{y})=\sum_{\ell=0}^{\infty} \sum_{\mathrm{k}=1}^{\mathrm{N}(\mathrm{n}, \ell)} \widehat{\phi}(\ell) \mathrm{Y}_{\ell, \mathrm{k}}(\boldsymbol{x}) \mathrm{Y}_{\ell, \mathrm{k}}(\mathbf{y})
$$

Chen et al. [2] proved that the kernel $\Phi$ is strictly positive definite if and only if $\widehat{\phi}(\ell) \geqslant 0$ for all $\ell \geqslant 0$, and $\widehat{\phi}(\ell)>0$ for infinitely many even values of $\ell$ and infinitely many odd values of $\ell$; this is also discussed by Schoenberg [9]. We construct a positive definite kernel on $\mathbb{S}^{n}$ by restricting a radial basis function $\varphi$ in $\mathbb{R}^{n+1}$ onto $\mathbb{S}^{n}$. As discussed by Wendland [10], $\varphi: \mathbb{R}^{n+1} \rightarrow \mathbb{R}$ is a radial basis function if: (i) $\varphi(\boldsymbol{x})=\rho(\|\boldsymbol{x}\|)$ for some univariate function $\rho$; and 
(ii) $\varphi$ is positive definite, that is, for any set of scattered points $\left\{\boldsymbol{x}_{1}, \ldots, \boldsymbol{x}_{\mathrm{K}}\right\} \subset$ $\mathbb{R}^{n+1}$, the matrix $\left[\varphi\left(\boldsymbol{x}_{i}-\boldsymbol{x}_{j}\right)\right]_{i, j=1}^{K}$ is positive definite. Wendland [10] provides more background on radial basis functions. We now define a positive definite kernel

$$
\Phi(\boldsymbol{x}, \boldsymbol{y})=\varphi(\boldsymbol{x}-\mathbf{y})=\rho(\|\boldsymbol{x}-\mathbf{y}\|)=\rho(\sqrt{2-2 \boldsymbol{x} \cdot \boldsymbol{y}}) \quad \text { for } \boldsymbol{x}, \boldsymbol{y} \in \mathbb{S}^{n}
$$

This means the function $\phi$ given in (4) is chosen to be $\phi(t)=\rho(\sqrt{2-2 t})$. In the numerical experiments, we choose $\rho(r)=(1-r)_{+}^{4}(1+4 r)$, where $(x)_{+}=x$ if $x>0$ and $(x)_{+}=0$ if $x \leqslant 0$.

\section{Ill-posedness of the backward heat equation on $\mathbb{S}^{n}$}

The backward heat problem on the unit sphere is ill-posed (in the sense of Hadamard [5]), as shown in the following proposition.

Proposition 1. Suppose f satisfies

$$
\sum_{\ell=0}^{\infty} e^{2 \lambda_{\ell} T} \sum_{k=1}^{N(n, \ell)}\left|\widehat{f}_{\ell, k}\right|^{2}<\infty .
$$

Then the problem (1) has the unique solution

$$
u(x, t)=\sum_{\ell=0}^{\infty} e^{\lambda_{\ell}(T-t)} \sum_{k=1}^{N(n, \ell)} \widehat{f}_{\ell, k} \gamma_{\ell, k}(x) .
$$

This solution does not depend continuously on the given data $\mathrm{f}$.

Proof: Consider the initial value problem

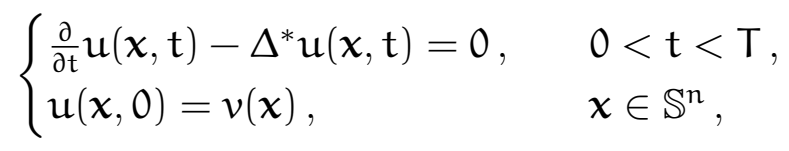


where $v$ is some given function. By the method of separation of variables, the solution to (7) is

$$
u(x, t)=\sum_{\ell=0}^{\infty} e^{-\lambda_{\ell} t} \sum_{k=1}^{N(n, \ell)} \widehat{v}_{\ell, k} Y_{\ell, k}(\boldsymbol{x}) .
$$

If $u$ is the solution of (1), then $u(x, T)=f(\boldsymbol{x})$ implies

$$
u(\boldsymbol{x}, T)=\sum_{\ell=0}^{\infty} e^{-\lambda_{\ell} \mathrm{T}} \sum_{\mathrm{k}=1}^{\mathrm{N}(\mathrm{n}, \ell)} \widehat{v}_{\ell, \mathrm{k}} \mathrm{Y}_{\ell, \mathrm{k}}(\boldsymbol{x})=\sum_{\ell=0}^{\infty} \sum_{\mathrm{k}=1}^{\mathrm{N}(\mathrm{n}, \ell)} \widehat{\hat{f}}_{\ell, \mathrm{k}} \mathrm{Y}_{\ell, \mathrm{k}}(\boldsymbol{x}),
$$

so that $\widehat{v}_{\ell, \mathrm{k}}=\mathrm{e}^{\lambda_{\ell} \mathrm{T}} \widehat{f}_{\ell, \mathrm{k}}$. So, it follows from (8) that the solution to (1) is

$$
u(x, t)=\sum_{\ell=0}^{\infty} e^{\lambda_{\ell}(T-t)} \sum_{k=1}^{N(n, \ell)} \widehat{f}_{\ell, k} Y_{\ell, k}(x)
$$

To prove that the solution (10) does not depend continuously on the given data $f$, let $f_{\ell}=Y_{\ell, 0} / \sqrt{\lambda_{\ell}}$ for $\ell=1,2, \ldots$ From (10), the solution to (1) when $f=f_{\ell}$ is

$$
u_{\ell}(x, t)=e^{\lambda_{\ell}(T-t)} \frac{1}{\sqrt{\lambda_{\ell}}} Y_{\ell, 0}(x) .
$$

It is trivial to see when $f=f_{0} \equiv 0$ the solution to the backward heat problem (1) is $\mathfrak{u}=\mathfrak{u}_{0} \equiv 0$. As $\ell \rightarrow \infty$,

$$
\left\|f_{\ell}-f_{0}\right\|_{L^{2}\left(\mathbb{S}^{n}\right)}^{2}=\frac{1}{\lambda_{\ell}} \rightarrow 0
$$

but

$$
\left\|u_{\ell}(\cdot, 0)-u_{0}(\cdot, 0)\right\|_{L^{2}\left(\mathbb{S}^{n}\right)}^{2}=\frac{e^{2 \lambda_{\ell} T}}{\lambda_{\ell}} \rightarrow+\infty
$$

So the solution does not depend continuously of the data $f$. 


\section{Regularization and approximation}

\subsection{The regularized problem}

Following a regularization technique for parabolic problems proposed by Clark and Oppenheimer [3], we consider the regularized problem

$$
\begin{cases}\frac{\partial}{\partial t} \mathfrak{u}^{\epsilon}(\boldsymbol{x}, \mathrm{t})-\Delta^{*} \mathfrak{u}^{\epsilon}(\boldsymbol{x}, \mathrm{t})=0, & 0<\mathrm{t}<\mathrm{T}, \\ \mathfrak{u}^{\epsilon}(\boldsymbol{x}, T)+\alpha(\epsilon) \mathfrak{u}^{\epsilon}(\boldsymbol{x}, 0)=f^{\epsilon}(\boldsymbol{x}), & \boldsymbol{x} \in \mathbb{S}^{\mathfrak{n}} .\end{cases}
$$

Here $\alpha(\epsilon)$ is a regularized parameter depending on some given parameter $\epsilon$ satisfying $0<\epsilon<1$, and $f^{\epsilon}$ is a perturbed version of $f$ so that $\left\|f^{\epsilon}-f\right\|_{L^{2}\left(\mathbb{S}^{n}\right)}<$ $M \epsilon$ with some fixed constant $M$. Clark and Oppenheimer [3] showed that the regularized problem is well-posed.

With the help of (11), we approximate $\mathfrak{u}(\boldsymbol{x}, 0)$ by $\mathfrak{u}^{\epsilon}(\boldsymbol{x}, 0)$, which is found by solving a pseudo-differential equation which is derived below. Let

$$
u^{\epsilon}(x, 0)=\sum_{\ell=0}^{\infty} \sum_{k=1}^{N(n, \ell)}{\widehat{v^{\epsilon}}}_{\ell, k} Y_{\ell, k}(x) \text {. }
$$

Then, from (8),

$$
\mathfrak{u}^{\epsilon}(\boldsymbol{x}, \mathrm{t})=\sum_{\ell=0}^{\infty} e^{-\lambda_{\ell} \mathrm{t}} \sum_{k=1}^{N(n, \ell)}{\widehat{v^{\epsilon}}}_{\ell, k} \gamma_{\ell, k}(\boldsymbol{x})
$$

implying

$$
u^{\epsilon}(\boldsymbol{x}, T)=\sum_{\ell=0}^{\infty} e^{-\lambda_{\ell} T} \sum_{k=1}^{N(n, \ell)}{\widehat{v^{\epsilon}}}_{\ell, k} Y_{\ell, k}(\boldsymbol{x})
$$

So, from the second equation of (11), by equating the Fourier coefficients, we obtain

$$
{\widehat{v^{\epsilon}}}_{\ell, k}\left[e^{-\lambda T}+\alpha(\epsilon)\right]=\widehat{f}_{\ell, k} \quad \text { or } \quad \widehat{v}_{\ell, k}=\left[e^{-\lambda T}+\alpha(\epsilon)\right]^{-1} \widehat{f}_{\ell, k} .
$$


Therefore, problem (11) has a unique solution which is given by the series

$$
u^{\epsilon}(\boldsymbol{x}, \mathrm{t})=\sum_{\ell=0}^{\infty} e^{-\lambda_{\ell} \mathrm{t}}\left[\alpha(\epsilon)+e^{-\lambda_{\ell} \mathrm{T}}\right]^{-1} \sum_{k=1}^{N(n, \ell)} \widehat{f}_{\ell, k} Y_{\ell, k}(\boldsymbol{x}) .
$$

At $t=0$,

$$
u^{\epsilon}(\boldsymbol{x}, 0)=\sum_{\ell=0}^{\infty}\left[\alpha(\epsilon)+e^{-\lambda_{\ell} \mathrm{T}}\right]^{-1} \sum_{k=1}^{N(n, \ell)} \widehat{f}_{\ell, k} Y_{\ell, k}(\boldsymbol{x}) .
$$

So if we define a pseudo-differential operator $L^{\epsilon}$ by

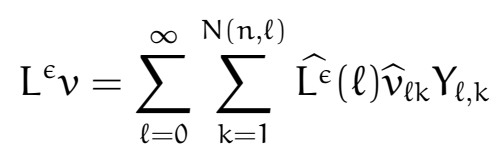

where $\widehat{\mathrm{L}^{\epsilon}}(\ell)=\alpha(\epsilon)+e^{-\lambda_{\ell} T}$, then

$$
\mathrm{L}^{\epsilon} \mathrm{u}^{\epsilon}(\boldsymbol{x}, 0)=\boldsymbol{f}^{\epsilon}(\boldsymbol{x}) .
$$

In the next sections we solve (14) numerically using spherical radial basis functions.

\subsection{Galerkin approximation to the regularized problem}

Let $X=\left\{\boldsymbol{x}_{1}, \ldots, \boldsymbol{x}_{\mathrm{N}}\right\}$ be a set of scattered points on the unit sphere and let $\Phi_{i}=\Phi\left(x_{i}, \cdot\right)$. We define the finite dimensional space

$$
\mathrm{V}_{\mathrm{X}}=\operatorname{span}\left\{\Phi_{i}: i=1, \ldots, \mathrm{N}\right\}
$$

The Galerkin approximation to the regularized problem is: find $\mathfrak{u}_{X}^{\epsilon} \in V_{X}$ so that

$$
\left\langle\mathrm{L}^{\epsilon} \mathfrak{u}_{X}^{\epsilon}, \Phi_{j}\right\rangle=\left\langle\mathrm{f}^{\epsilon}, \Phi_{j}\right\rangle \quad \text { for all } j=1, \ldots, N
$$


For $u_{X}^{\epsilon}=\sum_{j=1}^{N} c_{j} \Phi_{j}$ the coefficients $c=\left\{c_{j}: j=1, \ldots, N\right\}$ are determined by solving the linear system

$$
A \mathbf{c}=\mathbf{f} .
$$

Here, the elements of vector $\mathbf{f}$ are $\boldsymbol{f}_{j}=\left\langle\boldsymbol{f}^{\epsilon}, \Phi_{j}\right\rangle$ for $\boldsymbol{j}=1, \ldots, N$. The entries of the Galerkin matrix $A$ are

$$
\begin{aligned}
A_{i j} & =\left\langle L^{\epsilon} \Phi_{j}, \Phi_{i}\right\rangle \\
& =\sum_{\ell=0}^{\infty}[\widehat{\phi}(\ell)]^{2}\left[e^{-\lambda_{\ell} T}+\alpha(\epsilon)\right] \sum_{k=1}^{N(n, \ell)} Y_{\ell, k}\left(x_{i}\right) Y_{\ell, k}\left(x_{j}\right) \\
& =\sum_{\ell=0}^{\infty}[\widehat{\phi}(\ell)]^{2}\left[e^{-\lambda_{\ell} T}+\alpha(\epsilon)\right] \frac{N(n, \ell)}{\omega_{n}} P_{\ell}\left(x_{i} \cdot x_{j}\right) .
\end{aligned}
$$

\section{$5 \quad$ Numerical experiments}

We consider the backward heat problem (1) on the two-dimensional sphere $\mathbb{S}^{2}$, with $T=1$ and $f$ chosen so that the exact solution is

$$
u(x, t)=\sum_{\ell=1}^{\infty} \frac{e^{-\ell(\ell+1) t}}{\ell(\ell+1)} P_{\ell}(x \cdot p),
$$

where the $P_{\ell}$ are the Legendre polynomials and $\boldsymbol{p}$ is the north pole $(0,0,1)$. When $t=0$ there is a closed form

$$
u(x, 0)=\sum_{\ell=1}^{\infty} \frac{1}{\ell(\ell+1)} P_{\ell}(z)=1-2 \log [1+\sqrt{(1-z) / 2}], \quad \text { for } z=x \cdot p
$$

In the regularized problem (11) we take

$$
f^{\epsilon}(\boldsymbol{x})=\sum_{\ell=1}^{\infty} \frac{e^{-\ell(\ell+1)}+\alpha(\epsilon)}{\ell(\ell+1)} \mathrm{P}_{\ell}(\boldsymbol{x} \cdot \mathbf{p})
$$


Figure 1: Saff-Kuijlaars points $(\mathrm{N}=1500)$ indicated by red dots.

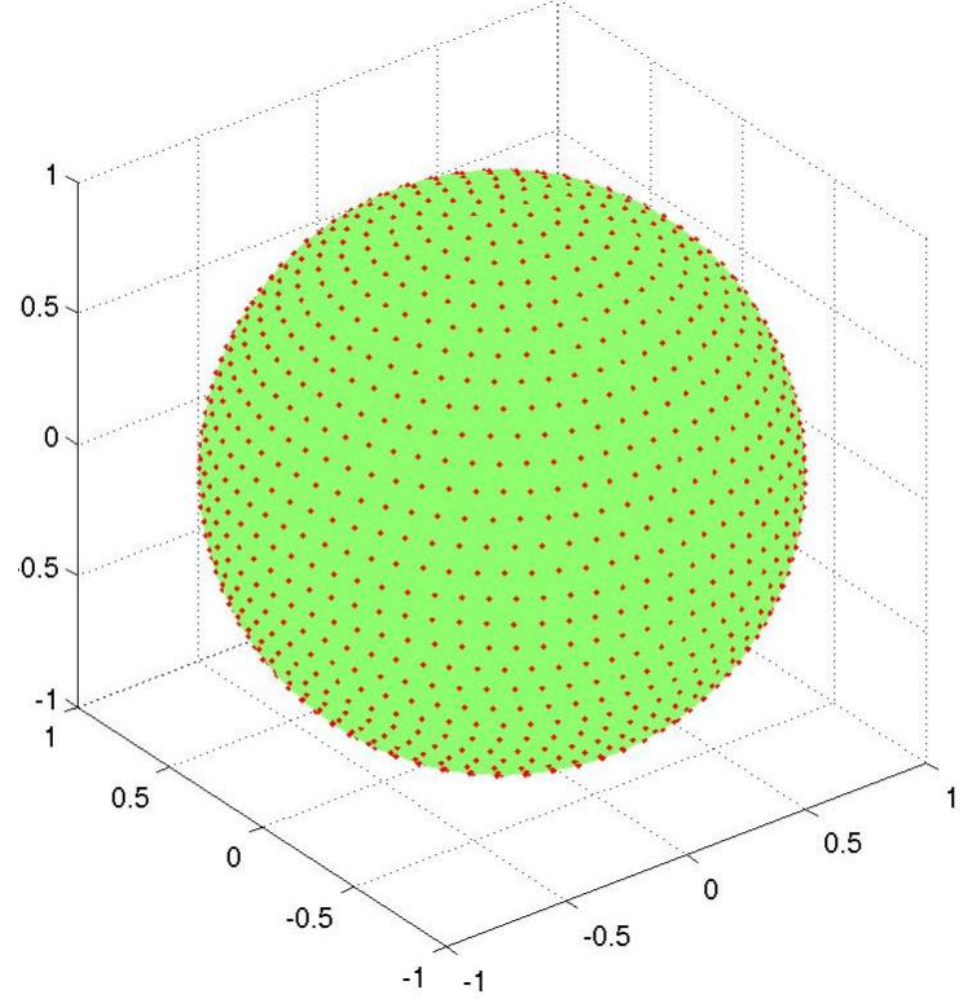

where $\alpha(\epsilon)=\epsilon$ in the first set of experiments and $\alpha(\epsilon)=\epsilon^{1 / 2}$ in the second set. For the Galerkin approximation problem (15) we choose the kernel

$$
\Phi(\boldsymbol{x}, \mathbf{y})=\rho(\sqrt{2-2 x \cdot y}) \quad \text { with } \rho(r)=(1-r)_{+}^{4}(1+4 r) .
$$

Figure 1 shows the set of points $X$ generated using the equal area partitioning algorithm of Saff and Kuijlaars [8]. Evolution of the temperature distributions are observed in Figures 2 and 3 where we plot the temperature at time $t=0$ (namely $u(x, 0)$ ) and at time $t=1$ (perturbed with $\epsilon=10^{-2}$ ). 
Figure 2: Exact temperature distribution $u(x, 0)$ at time $t=0$.
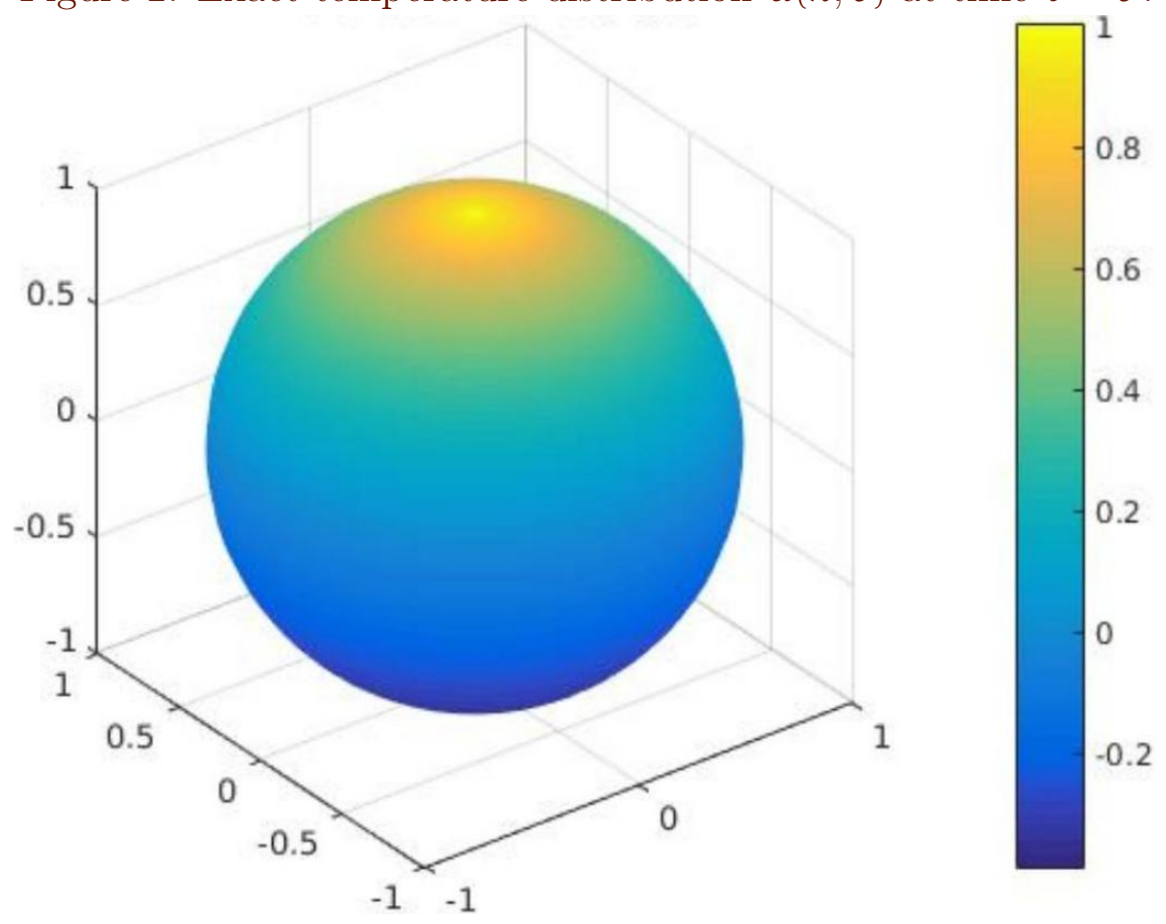

We compute the right hand side of (15) using

$$
\left\langle\mathrm{f}^{\epsilon}, \Phi_{j}\right\rangle=\sum_{\ell=1}^{\infty} \frac{e^{-\ell(\ell+1)}+\alpha(\epsilon)}{\ell(\ell+1)} \widehat{\phi}(\ell) \mathrm{P}_{\ell}\left(\boldsymbol{x}_{\mathbf{j}} \cdot \mathbf{p}\right) .
$$

The one-dimensional integral

$$
\widehat{\phi}(\ell)=2 \pi \int_{-1}^{1} \phi(t) P_{\ell}(t) d t
$$

is a direct consequence of (4) when $n=2$ and the orthogonality of the Legendre polynomials. 
Figure 3: Temperature distribution $\mathrm{f}^{\epsilon}$ at time $t=1$ perturbed with $\epsilon=10^{-2}$.
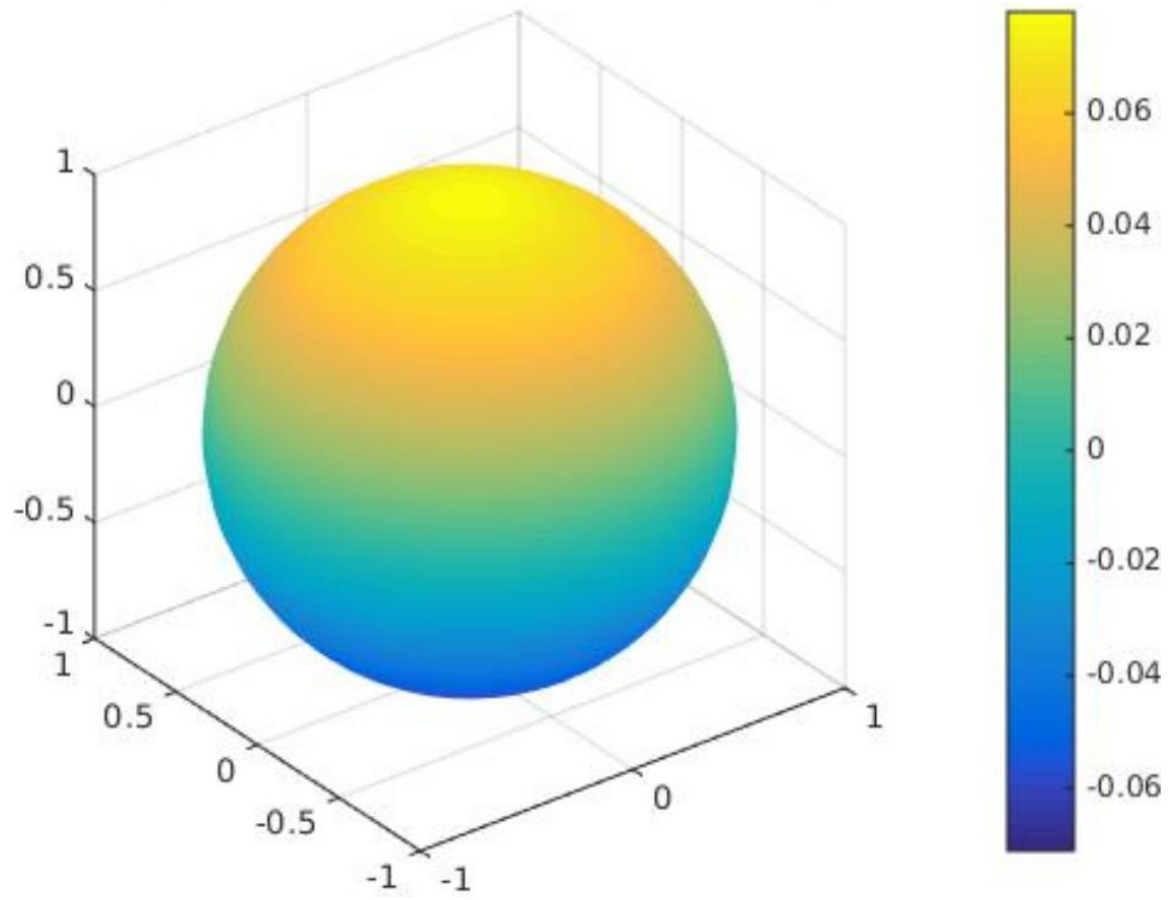

The numerical results are summarized in Figures 4 and 5 . The discrete $\ell_{2}$ errors are computed using

$$
\|e\|_{\ell_{2}}=\left(\frac{1}{|\mathcal{G}|} \sum_{\xi \in \mathcal{G}}\left|u_{X}^{\epsilon}(\xi, 0)-u(\xi, 0)\right|^{2}\right)^{1 / 2}
$$

where $\mathcal{G}$ is a set of 19000 random scattered points on $\mathbb{S}^{2}$.

In Figure 4 with $\epsilon=10^{-2}$, as the number of Saff-Kuijlaars points $N$ increases, the approximation errors decrease. In Figure 5 we choose $\alpha=\epsilon=10^{-5}$ and the errors stabilize when $N \geqslant 400$. The numerical results suggest that when the parameter $\alpha(\epsilon)$ is too close to zero the problem is close to ill-posedness, resulting in a poor approximation. 
Figure 4: Approximation of $\ell_{2}$ errors using $\epsilon=10^{-2}$.

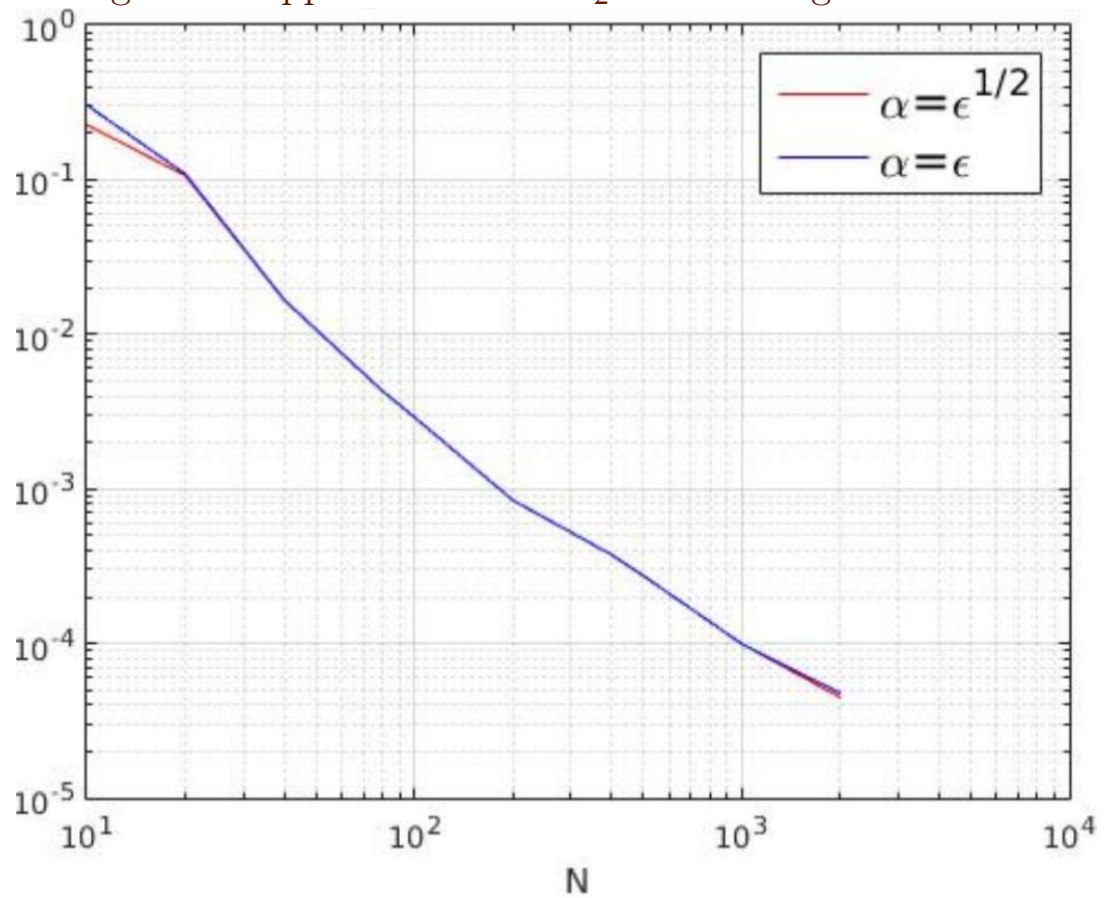

\section{Conclusion}

We showed formally that the backward heat problem on the unit sphere is ill-posed. A regularized strategy is proposed and the problem is converted to a pseudo-differential equation. A Galerkin approximation scheme to the pseudodifferential equation using spherical radial basis function was developed. Convergence analysis of the numerical approach and suitable choices of the regularized parameter $\alpha(\epsilon)$ are the subjects of a future publication.

Acknowledgement This work was initiated while Huy Tuan Nguyen was visiting the School of Mathematics and Statistics, UNSW. His visit was 


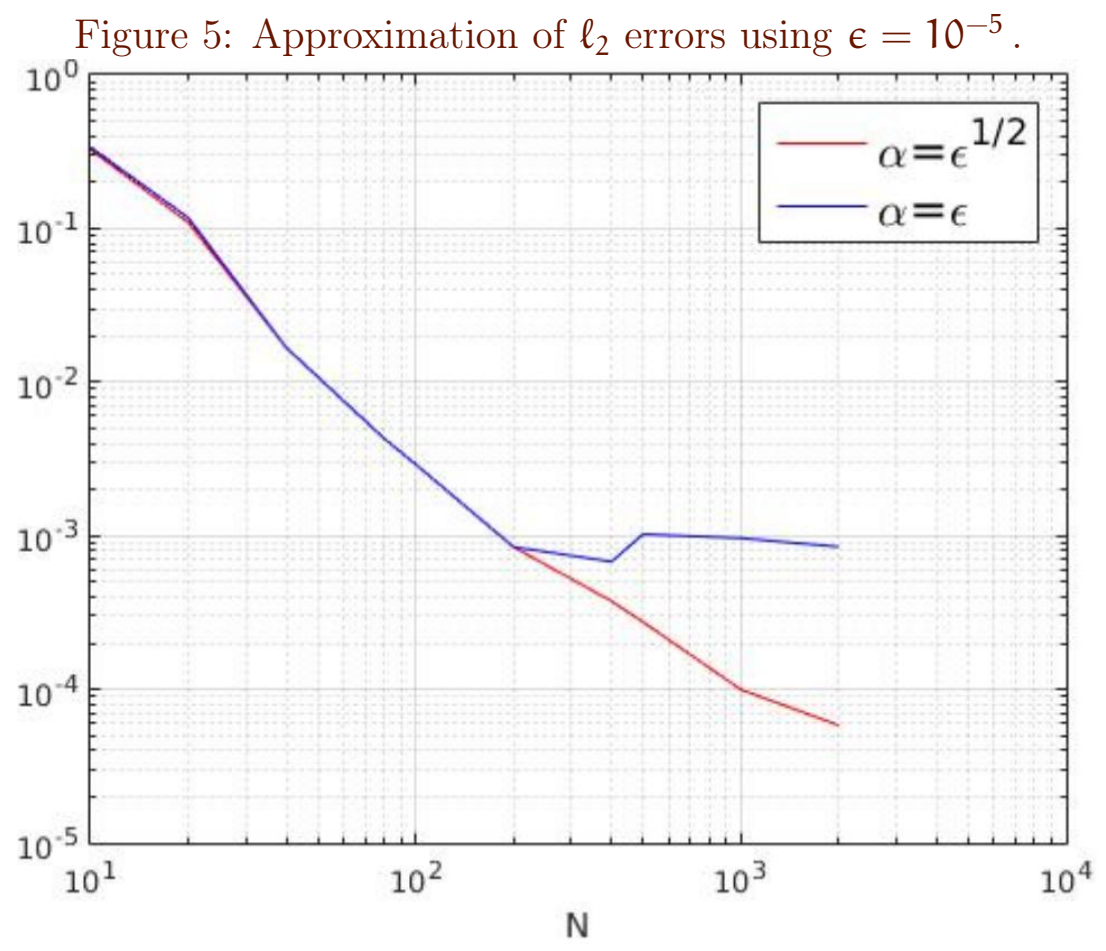

partially supported by the Australian Research Council Grant DP 120101886.

\section{References}

[1] J. Atmadja and A. C. Bagtzoglou. Pollution source identification in heterogeneous porous media. Water Resour. Res., 37:2113-2125, 2001. doi:10.1029/2001wr000223 C263

[2] D. Chen, V. A. Menegatto and X. Sun. A necessary and sufficient condition for strictly positive definite functions on spheres. P. Am. Math. Soc., 131:2733-2740, 2003. doi:10.1090/S0002-9939-03-06730-3 C266 
[3] G. W. Clark and S. F. Oppenheimer. Quasireversibility methods for non-well-posed problems. Electron. J. Diff. Eq., 1994(8):1-9, 1994. http://ejde.math.unt.edu C264, C269

[4] I. G. Enting. Inverse problems in atmospheric constituent transport. Cambridge University Press, Cambridge, 2002. doi:10.1017/cbo9780511535741 C263

[5] J. Hadamard. Sur les problèmes aux dérivées partielles et leur signification physique. Princeton Uni. Bull., 13:49-52, 1902. C263, C267

[6] L. S. G. Kovasznay and H. M. Joseph. Image processing. Proc. IRE, 43:560-570, 1955. doi:10.1109/jrproc.1955.278100 C263

[7] C. Müller. Spherical Harmonics, volume 17 of Lecture Notes in Mathematics. Springer-Verlag, Berlin, 1966. doi:10.1007/bfb0094775 C265, C266

[8] E. B. Saff and A. B. J. Kuijlaars. Distributing many points on a sphere. Math. Intelligencer, 19:5-11, 1997. doi:10.1007/BF03024331 C272

[9] I. J. Schoenberg. Positive definite function on spheres. Duke Math. J., 9:96-108, 1942. doi:10.1215/S0012-7094-42-00908-6 C265, C266

[10] H. Wendland. Scattered Data Approximation. Cambridge University Press, Cambridge, 2005.

http://www. cambridge.org/au/academic/subjects/mathematics/ numerical-analysis/scattered-data-approximation C266, C267

\section{Author addresses}

1. Q. T. Le Gia, School of Mathematics and Statistics, The University of New South Wales, Sydney 2052, Australia

mailto:qlegia@unsw.edu .au 
2. Huy Tuan Nguyen, Department of Mathematics and Informatics, HCMC University of Science, Vietnam National University, Vietnam. mailto:thnguyen2683@gmail.com

3. Thanh Tran, School of Mathematics and Statistics, The University of New South Wales, Sydney 2052, Australia

mailto: thanh. tran@unsw. edu. au 